\title{
Diffusion tensor imaging and electrophysiology as robust assays to evaluate the severity of acute spinal cord injury in rats
}

Beike Chen ${ }^{1}$, Qiang Tan², Weikang Zhao ${ }^{1}$, Qiming Yang ${ }^{1}$, Hongyan Zhang ${ }^{2}$, Fabao Gao ${ }^{3}$, Xin Liu², Hua Feng ${ }^{2}$ and Dianming Jiang ${ }^{1,4^{*}}$

\begin{abstract}
Background: Diffusion tensor imaging (DTI) is an effective method to identify subtle changes to normal-appearing white matter (WM). Here we analyzed the DTI data with other examinations, including motor evoked potentials (MEPs), histopathological images, and behavioral results, to reflect the lesion development in different degrees of spinal cord injury (SCl) in acute and subacute stages.

Method: Except for 2 Sprague -Dawley rats which died from the anesthesia accident, the rest 42 female rats were randomized into 3 groups: control group $(n=6)$, moderate group $(n=18)$, and severe group $(n=18)$. Moderate (a 50-g aneurysm clip with 0.4-mm thickness spacer) or severe (a 50-g aneurysm clip with no spacer) contusion SCl at T8 vertebrae was induced. Then the electrophysiological assessments via MEPs, behavioral deterioration via the Basso, Beattie, and Bresnaha (BBB) scores, DTI data, and histopathology examination were analyzed.

Results: In this study, we found that the damage of WM myelin, MEPs amplitude, BBB scores and the decreases in the values of fractional anisotropy (FA) and axial diffusivity (AD) were more obvious in the severe injury group than those of the moderate group. Additionally, the FA and AD values could identify the extent of $S C I$ in subacute and early acute $\mathrm{SCI}$ respectively, which was reflected in a robust correlations with MEPs and BBB scores. While the values of radial diffusivity (RD) showed no significant changes.

Conclusions: Our data confirmed that DTI was a valuable in ex vivo imaging tool to identify damaged white matter tracts after graded $\mathrm{SCl}$ in rat, which may provide useful information for the early identification of the severity of $\mathrm{SCl}$.
\end{abstract}

Keywords: Anisotropy, DTI, SCI, MEPS, BBB, White matter, Neuroimaging

\footnotetext{
* Correspondence: 201296@hospital.cqmu.edu.cn

'Department of Orthopedics, The First Affiliated Hospital of Chongqing

Medical University, Chongqing 400016, People's Republic of China

${ }^{4}$ Department of Orthopedics, The Third Affiliated Hospital of Chongqing

Medical University (Gener Hospital), Chongqing 401120, People's Republic of

China

Full list of author information is available at the end of the article
}

(c) The Author(s). 2020 Open Access This article is licensed under a Creative Commons Attribution 4.0 International License, which permits use, sharing, adaptation, distribution and reproduction in any medium or format, as long as you give appropriate credit to the original author(s) and the source, provide a link to the Creative Commons licence, and indicate if changes were made. The images or other third party material in this article are included in the article's Creative Commons licence, unless indicated otherwise in a credit line to the material. If material is not included in the article's Creative Commons licence and your intended use is not permitted by statutory regulation or exceeds the permitted use, you will need to obtain permission directly from the copyright holder. To view a copy of this licence, visit http://creativecommons.org/licenses/by/4.0/ The Creative Commons Public Domain Dedication waiver (http://creativecommons.org/publicdomain/zero/1.0/) applies to the data made available in this article, unless otherwise stated in a credit line to the data. 


\section{Background}

Early intervention in acute stage of spinal cord injury (SCI) can reduce the adverse effect of glial scar and cavity on axon regeneration, showing a superiority in spinal cord repair [1]. Timely and effective identification of SCI severity, which provides important information and therefore affects early clinical decision-making, is the key to early intervention [2,3]. The identification of the severity of SCI commonly depends on the International Standards for the Neurological Classification of Spinal Cord Injury $[4,5]$. However, since the neurological responses can be affected by the spinal shock in acute stage of SCI, the reliability of current standards, which is based on the strength of various muscles as well as neurological responses to sensory input on both sides of the body, for early identification of the severity of SCI is limited [6].

Currently, magnetic resonance imaging (MRI) is the main imaging modality to visualize the injury characteristics of SCI [7]. However, since the integrity of white matter tract is the main factor affecting the function outcome of SCI patients, and conventional T2 weighted images is not suitable for the evaluation of white matter tracts, the utility of anatomic MRI as a prognostic tool is limited during the acute phase of SCI [8]. Diffusion tensor imaging (DTI), weighted with the local microstructural characteristics of water diffusion, provides an effective means of visualizing functional connectivity in the nervous system, and is being used to identify subtle changes to the normal-appearing white matter on anatomic MRI [9-11]. As a noninvasive method, DTI also showed superiority on differentiation of low-grade from high-grade gliomas and residual/recurrent gliomas from post-radiation changes $[12,13]$. Although the changes of spinal cord after acute injury has been observed by DTI, the relationship between these changes and the degree of injury is unclear.

Electrophysiology, such as motor evoked potentials (MEPs), is a good method to evaluate the functional integrity of spinal pathways [14], and has been used to identify connected but nonfunctional axons crossing SCI lesions in rodents [15], dogs [16], and non-human primates [17]. The MEPs amplitude in rat SCI model has been reported to be positively correlated with the preserved tissue at the injured site $[18,19]$. The permeability of the axonal membrane to water is intimately tied to MR diffusion measurements [20], as well as the morphometry tied to neuronal excitability [21]. Therefore, it is reasonable to assume that the changes in DTI measurements is correlated with the MEP parameters.

Accordingly, the consistency and capability of DTI to reflect the lesion development, as well as the relationship between imaging and other examinations in different degrees of SCI in acute stage, was explored in a most commonly used rat model.

\section{Methods}

\section{Ethic oversight}

Animal experimental procedures were performed under the Regulations for the Administration of Affairs Concerning Experimental Animals approved by the State Council of People's Republic of China. All experiments were conducted in accordance with animal care guidelines approved by the Laboratory Animal Welfare and Ethics Committee of Third Military Medical University (the Army Military Medical University).

\section{Study design and subjects}

All experiments were performed in the central laboratory in Southwest Hospital except for the ex vivo DTI acquisition and analyses, which were performed in the laboratory of department of Molecular Imaging in West China Hospital.

Adult female Sprague-Dawley (SD) rats (aged approximately three-months-old, 200-250 g) were obtained from the Army Military Medical University. Animals were housed in a controlled pathogen-free environment at a temperature of $23 \sim 25^{\circ} \mathrm{C}, 70 \%$ humidity, 12 h light-dark cycles, free access to food and water.

The sample size was calculated according to the previous study [22]. Except for 2 rats died from anesthesia accident, the rest 42 rats were randomized into 3 groups: control group $(n=6)$, moderate group $(n=18)$, and severe group $(n=18)$. Six rats from each group were tested for Basso, Beattie, and Bresnaha (BBB) score and MEPs test at different timepoints to evaluate the graded $\mathrm{SCI}$ injury in rats, and then animals were sacrificed on day 14 for ex vivo DTI and histological examination to provide imaging evidences. The rest animals from both moderate and severe group were sacrificed on day $3 / 7$ ( $n=6$ /group at each timepoint) for ex vivo DTI and histological examination. The euthanasia was performed with an intraperitoneal injection of $1 \%$ sodium pentobarbital overdoses (100 mg/kg body weight).

\section{$\mathrm{SCl}$ model}

SCI was induced according to the previous method [23, 24]. Briefly, rats were anesthetized with $1 \%$ sodium pentobarbital ( $40 \mathrm{mg} / \mathrm{kg}$ body weight, i. p.), and fixed in a prone position to perform a dorsal laminectomy at the T7-9vertebrae. A $50-\mathrm{g}$ aneurysm clip was used to laterally compress the T8 spinal cord with no spacer for $30 \mathrm{~s}$ to establish the severe injury model, while adding a 0.4-mm thickness spacer to establish the moderate injury model. The control group received identical laminectomy without crush injury. The skin incision was then closed. SCI rats were isolated to a separate cage in the animal house. Antibiotics (Cefazolin, $50 \mathrm{mg} / \mathrm{kg}$ body weight, i. p.) were given daily to treat/prevent bladder infection immediately after the surgery for 7 
continuous days. Bladders were pressed twice daily until the rats recovered to normal spontaneous micturition.

\section{Behavioral assessment of motor function}

All animals were assessed in an open-field walking according to the BBB locomotor scale [25], the day before inducing injury, and on day $1 / 3 / 7 / 14$ after operation. Hindlimb function was assessed according to the $0-21$ $\mathrm{BBB}$ scoring, where 0 is flaccid paralysis and 21 is normal gait.

\section{Electrophysiological assessments of hindlimb motor function}

MEPs were induced according to the previous method $[26,27]$. Briefly, rats were anesthetized with $1 \%$ pentobarbital sodium $(20 \mathrm{mg} / \mathrm{kg}$ i.p.). The stimulation needle electrode (DSN1620, Medtronic, USA), acting as the anode, was inserted subcutaneously at the base of the nose with the tip point touching the scalp. The needle electrode acting as the cathode was placed subcutaneously at the midpoint of the two ears. The recorded electrode was inserted into the tibialis anterior (TA) muscle. A ground electrode was placed subcutaneously at the base of the tail. A single pulse of electrical stimulation $(10 \mathrm{~mA}, 0.1 \mathrm{~ms}, 1 \mathrm{~Hz})$ to excite the brain was delivered via stimulator (Keypoint, Medtronic, USA). The electrical stimulation was repeated five times at an interval of $15 \mathrm{~s}$ in each rat. The base-to-peak amplitude of the trace after single stimulation was recorded for each type of evoked potential.

\section{Histology}

Rats were sacrificed at 3 days (3d), 7 days (7d) and 14 days (14d) post SCI. The spinal cords were harvested, fixed, dehydrated, embedded, and sliced into $20 \mu \mathrm{m}$ successively. After blocked with $5 \%$ bovine serum albumin (BSA) for $1.5 \mathrm{~h}$ at room temperature, the slices were incubated with the primary antibodies of goat anti-myelin basic protein (anti-MBP; 1:250; Santa Cruz; Cat: sc-13, 914) overnight at $4{ }^{\circ} \mathrm{C}$, and were probed subsequently with AlexaFluor-488-conjugated secondary antibodies against goat (1:200; Invitrogen) antibodies for $2 \mathrm{~h}$ at $37^{\circ} \mathrm{C}$. The nuclei were counterstained with 4 -6-diamidino-2-phenylindole (DAPI; Santa Cruz Biotechnology). Sections were imaged under a Zeiss confocal microscope (Zeiss, LSM780).

\section{Ex vivo DTI acquisition}

Magnetic resonance imaging was performed with a 7.0 Tesla Bruker Biospec 70/30 USR preclinical scaner, using a volume coil with a $23-\mathrm{mm}$ inner diameter and 44-mm outer diameter for both transmission and reception. The spinal cord specimens were loaded into $2 \mathrm{~mL}$ syringes filed with Fomblin (Solvay, Brussels, Belgium)
(28) and stabilized by a parallel plastic rod (6-mm diameter). RARE T2-weighted scans were acquired to locate the epicenter of the injury. Fifteen thick slices arranged around the epicenter were acquired for diffusion tensor imaging (sequence parameters: spin echo DTI-EPI, $b=$ $1319 \mathrm{~s} / \mathrm{mm}^{2}, \delta=4 \mathrm{~ms}, \Delta=18 \mathrm{~ms}, 30$ diffusion sampling directions, 16 averages, TE/TR $=32 / 2750 \mathrm{~ms}, 100 \mu \mathrm{m}$ inplane resolution, acquisition time $=3 \mathrm{~h} 6 \mathrm{~min} 40 \mathrm{~s}$ ).

Paravision Version 5.0 (Bruker BioSpin) was used for the analyses of B0, fractional anisotropy (FA) and mean diffusivity (MD) map. The cross-sectional areas of the spinal cord were defined by manually outlining the white matter (WM) areas on transverse B0 and FA scans for each time point. Of the 15 diffusion axial images acquired, the 11 axial slices centered on the lesion core were used for subsequent. Regions of interest (ROIs), which chose the total WM were manually outlined on the transverse images.

The DTI metrics including $\lambda 1, \lambda 2, \lambda 3, \mathrm{MD}$, and FA were used with the Paravision Version 5.0 (Bruker BioSpin, Karlsruhe, Germany) diffusion tensor calculation module according to the previous study [28]. The axial diffusivity $(\mathrm{AD})$ is a measure of the diffusivity along the principal axis of the diffusion tensor $(\mathrm{AD}=\lambda 1)$. The radial diffusivity $(\mathrm{RD})$ was calculated using the two minor diffusion axes $(R D=(\lambda 2+\lambda 3) / 2)$. The FA was calculated using the equation: $F A=\sqrt{ } 1 / 2\left(\sqrt{ }\left((\lambda 1-\lambda 2)^{2}+(\lambda 1\right.\right.$ $\left.\left.-\lambda 3)^{2}+(\lambda 2-\lambda 3)^{2}\right) / \sqrt{ }\left(\lambda 1^{2}+\lambda 2^{2}+\lambda 3^{2}\right)\right)$.

Fibertracks were visualised and analyzed using TrackVis (v. 0.6.1) by drawing ROIs on epicentral mask in ex vivo B0 maps for ROI filers. Then count the number of tracks which were greater than $3 \mathrm{~mm}$ was counted.

\section{Statistical analysis}

All DTI metrics were presented as mean and standard error of the mean (SEM). BBB score, MEPs amplitude and number of tracks were presented as mean and standard deviation (SD). BBB score, MEPs and DTI metrics data were analyzed using one-way analysis of variance (ANOVA), and group differences were ascertained using Tukey's post hoc comparisons when appropriate. Comparisons between 2 groups were analyzed using 2tailed Student t-tests. DTI metrics (including FA, AD and $\mathrm{RD}$ ) of the epicentral spinal cord were analyzed relatively to BBB and MEPs via Pearson's correlation. All statistical analyses were performed via the SPSS 23.0 software. The significance level for all tests were set at $P<0.05$.

\section{Result}

Graded SCI led to changes of locomotor capacity and MEPs amplitude

All rats were tested for locomotor capacity using the BBB locomotor scale. On day 1, both moderate and 
severe group showed nearly complete paralysis, which was reflected in a BBB score of $0 \sim 2$ (Fig. 1a). Then the animal's locomotor capacity began to recover gradually. On day 7 and 14 , the BBB scores of the moderate group were both higher than those of the severe group $(P<0.05)$, which indicated a better functional recovery in the moderate group. After the first day of SCI, both moderate and severe group exhibited a markedly reduced amplitude in MEPs. Then, the amplitude of MEPs rebounded gradually to that in second week (Fig. 1b). On day 7 and 14, the severe group had a lower MEPs amplitude than the moderate group (moderate vs severe, $P<0.05$, Fig. $1 \mathrm{~b}$ and $\mathrm{c}$ ), which suggested that the abnormal MEPs, as well as the central conduction, can still transmit through the spinal cord in relation to the severity of the injury.

Graded SCI was reflected by DTI-based morphometrics and metrics from the ventrolateral white matter (VWM) area The variations of the DTI metrics from the VWM area and typical B0, FA and MD maps from the epicenter towards the rostral and caudal were showed in Fig. 2. The values of
FA and $\mathrm{AD}$ at the epicenter decreased significantly after SCI (moderate, severe vs control, $P<0.05$ ). Compared with the moderate group, the severe group exhibited a more significant decline in FA value and $\mathrm{AD}$ value on day $7 / 14$ and $3 / 7$ respectively (moderate vs severe, $P<0.05$ ). The RD value in different positions of both moderate and severe groups were distributed in peak shape, and the value in the epicenter at all time points were similar $(P>0.05)$.

\section{Graded SCl on diffusion tensor tractography}

Based on the rat in ex vivo diffusion tensor data, we created fibertracks to depict the diffusion tensor tract fibers (DTT) of the white matter through the epicenter. In the DTT, each path was described in blue colour according to its superior-inferior (rostral-caudal) diffusion direction. In the white matter of normal spinal cord, we observed compact and orderly blue fiber tracts (Fig. 3a). The number of tracks across the epicenter of the white matters in the moderate groups was more than that in the severe groups (Fig. $3 b)$ on day 7 and $14(P<0.05)$, but similar on day $3(P>0.05)$.
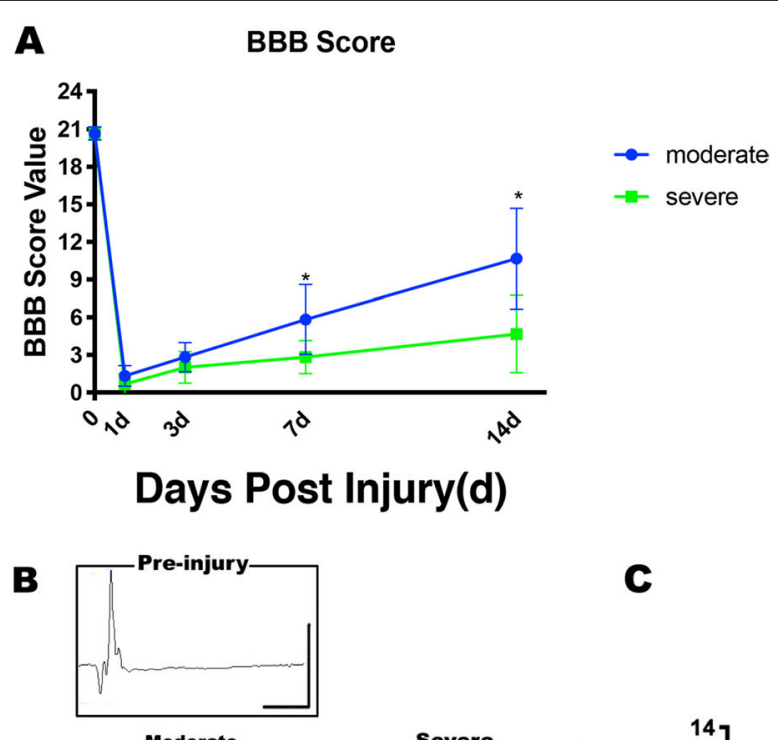

C
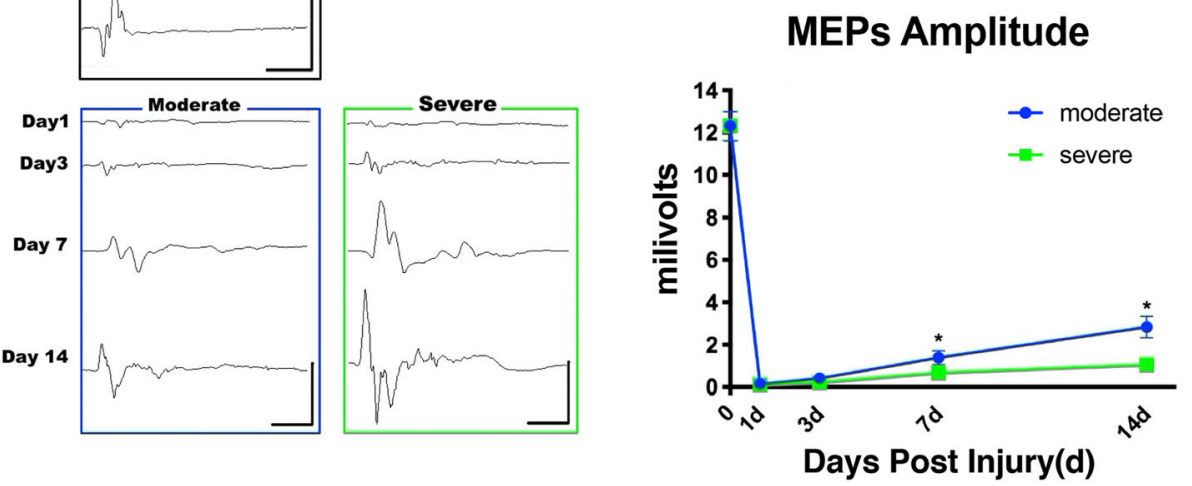

Fig. 1 Hindlimb motor function after different severities of SCI. a The BBB scores was similar between the moderate group and severe group on day 1 to day 3, but reached a significant difference from day 7 to day 14 (6 rats/group). b Representative recording of MEPs from control group, moderate and severe groups at various time points. c The MEPs was similar between the moderate group and severe group on day 1 to day 3 , but reached a significant difference from day 7 to day 14 ( 6 rats/group). Results are presented as mean \pm SD, ${ }^{*} P<0.05$. Scale: $5 \mathrm{mV} / 10 \mathrm{~ms}$ in preinjury recordings, and $2 \mathrm{mV} / 10 \mathrm{~ms}$ at other time points 


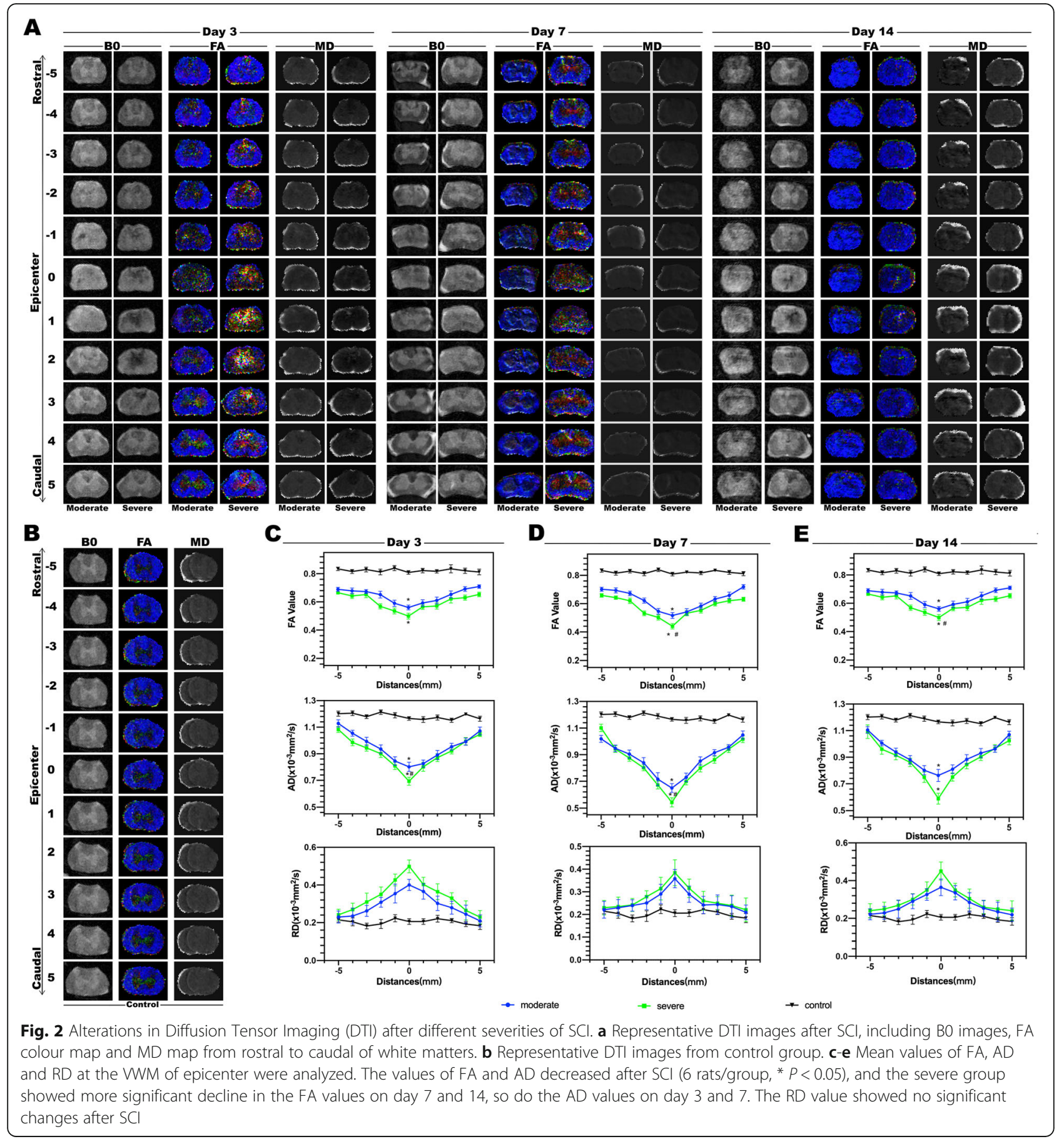

The effect of graded $\mathrm{SCl}$ on the integrity of white matter myelin

In the control group, Myelin Basic Protein (MBP) immunofluorescence was visualized as the characteristic ring in the VWM area. After injury, complete rings of myelin could not be seen at the same areas of epicenter (Fig. 4a-g), which suggested that the MBP fluorescence intensity decreased and myelin was markedly deteriorated in relation to the severity of injury.
Correlation between DTI metrics and locomotor capacity /MEPs amplitude after SCI

Correlation analyses demonstrated that the BBB scores were significantly correlated with epicentral FA values of the VWM area at day $7(r=0.790 ; P<0.01)$ and $14(r=$ 0.767; $P<0.01$; Fig. 5a, b), as well as the epicentral AD values of the VWM area at day $7(r=0.741 ; P<0.01)$ and $14(r=0.616 ; P<0.05$; Fig. $5 \mathrm{c}, \mathrm{d})$. Similarly, MEPs were significantly correlated with epicentral FA values of 

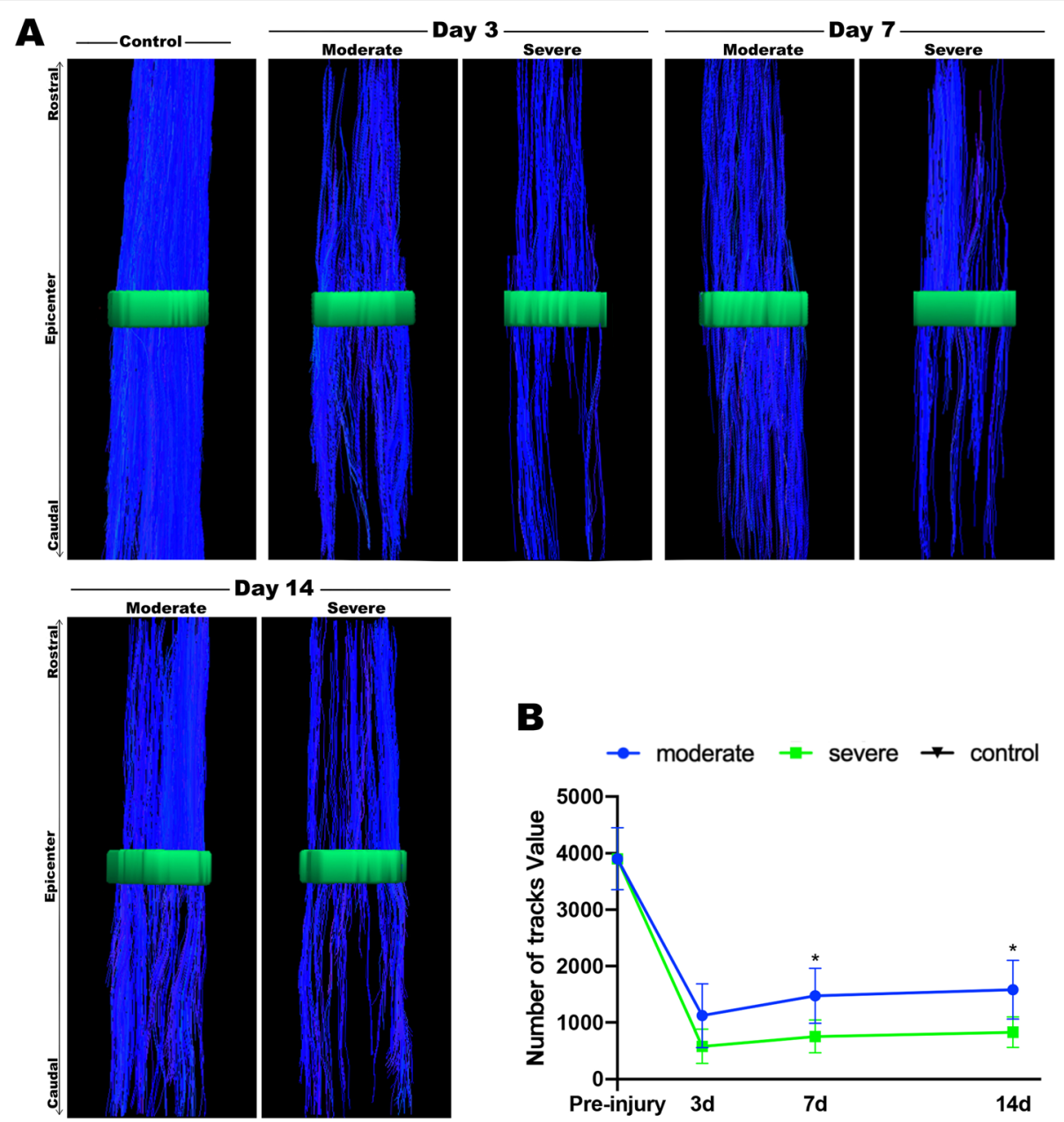

B

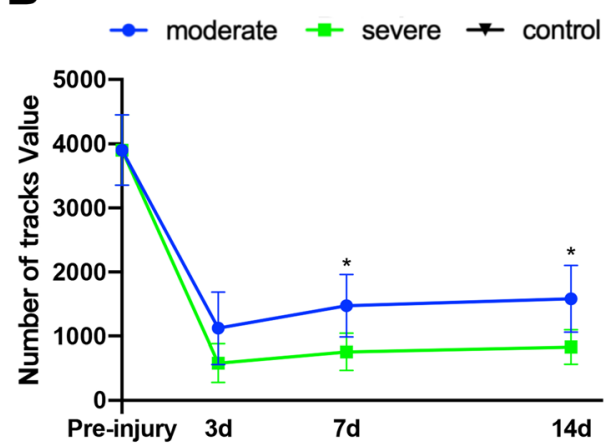

Fig. 3 White matters tracks detected by DTT (diffusion tensor tractography) after different severities of SCl. a Representative DTT images. The green ROls were the epicenter of white matters. White matters fibers were represented by blue lines. $\mathbf{b}$ Quantitation of white matters tracks across the ROls. Results are presented as mean \pm SD, ${ }^{*} P<0.05$

the VWM area at day $7(r=0.874 ; P<0.01)$ and $14(r=$ $0.792 ; P<0.01$; Fig. $6 a, b)$, as well as, epicentral AD values of the VWM area at day $7(r=0.781 ; P<0.01)$ and $14(r=$ $0.735 ; P<0.01 ;$ Fig. $6 c, d)$. This shows the capacity of FA and $\mathrm{AD}$ values to accurately assess motor function.

\section{Discussion}

In this study, we used ex vivo diffusion tensor imaging to assess the graded severities of contusion SCI at different time points and analyzed the correlation between imaging results and corresponding electrophysiological and behavioral outcome. The results suggested that DTI might be an important noninvasive technique to diagnose the severity of acute and subacute SCI, as well as a tool to evaluate functional changes. The $\mathrm{AD}$ and FA values were the most robust diffusion MRI indicator of damage in early acute and subacute SCI, respectively. Additionally, FA in VWM area showed a most robust correlation with MEP and BBB score at day 7. To our best knowledge, it is the first time that the correlation between the MEP and DTI metrics after acute SCI has been explored.

The conventional MRI, like T2WI, is regard as a useful modality for the visual assessment of spinal cord [29]. However, it is not suitable for identifying the integrity of WM tracts in acute SCI [8]. Diffusion-weighted MR imaging was sensitive to identify the structural changes or/ and edema grade differences in human diseases, like gliomas [12, 13], paraspinal neurogenic tumor [30], pathologic fracture [31] and SCI [32]. By correlating the diffusion-weighted MR imaging metrics with biopsy results of the mediastinal neurogenic tumor, a retrospective analysis found that the ADC values could be used to differentiate malignant from benign neurogenic tumors and schwannomas from neurofibromas with a high accuracy and sensitivity [30]. Different diffusion MRI metrics mean different in SCI: reduced FA indicates the demyelination of spared axon and axonal damage, as well as decreased AD means axonal collapse [33, 34], 

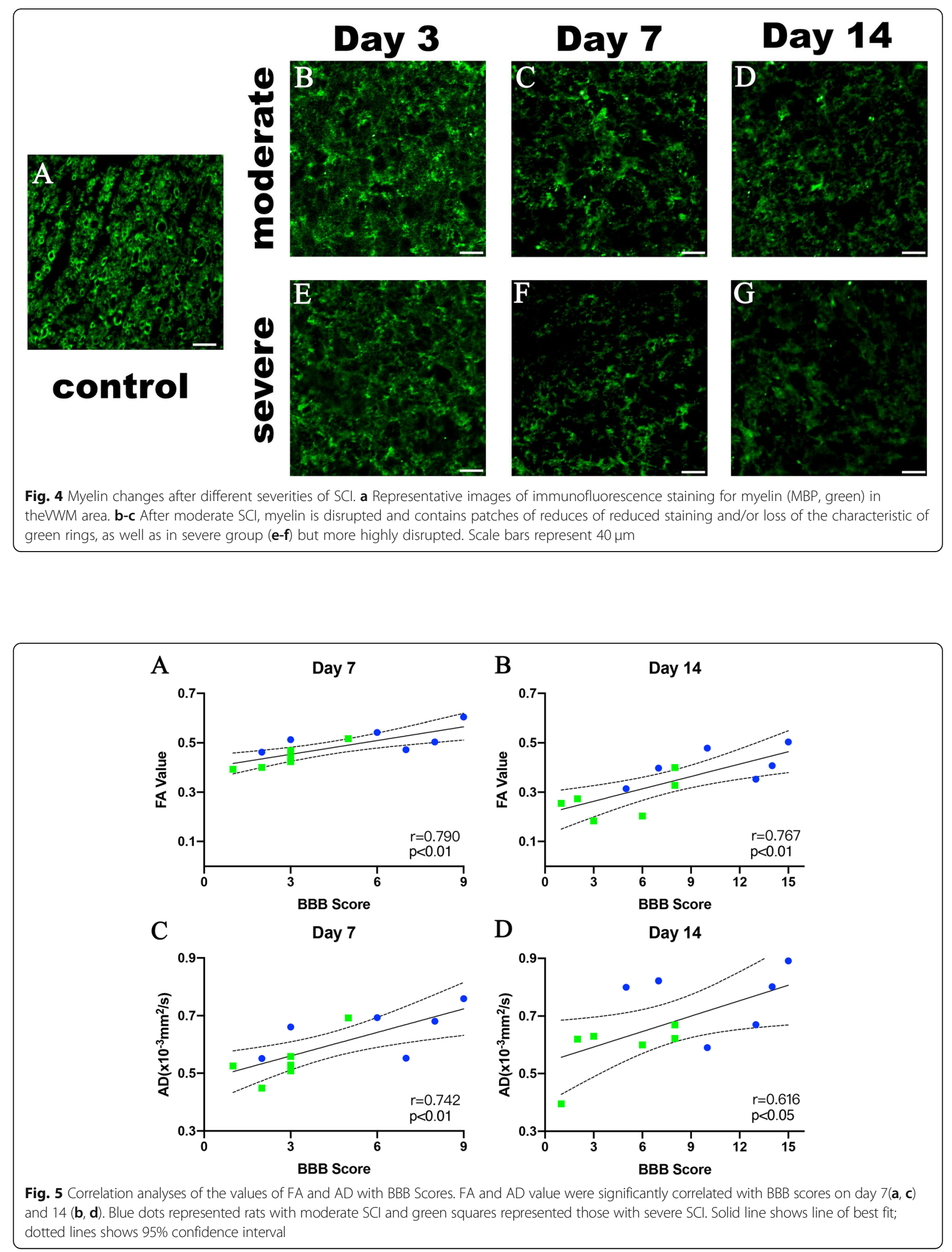

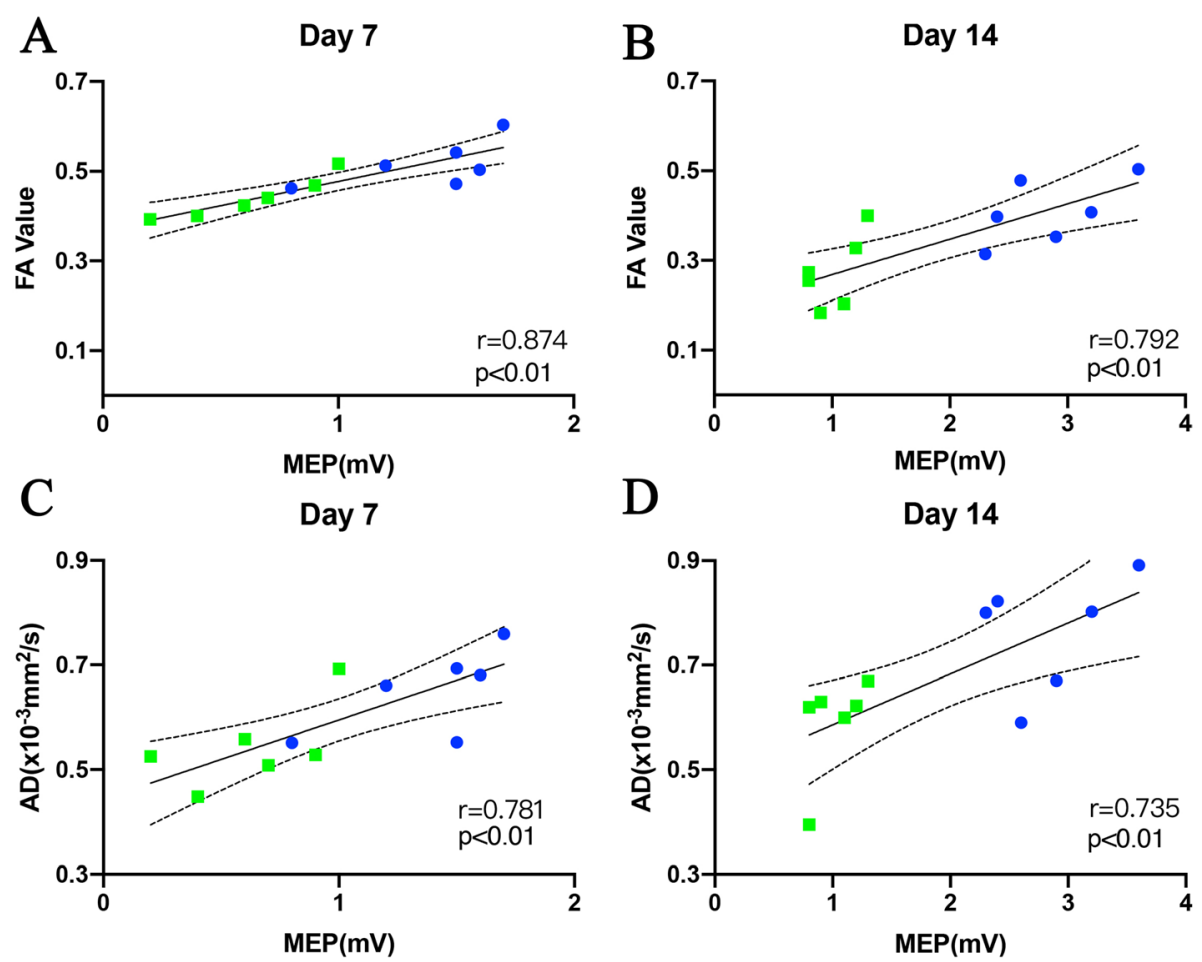

Fig. 6 Correlation analyses of the values of FA and AD with MEPS. FA and AD value were significantly correlated with MEPs on day 7(a, c) and 14 (b, d). Blue dot represented rats with moderate $\mathrm{SCl}$ and green square represented those with severe $\mathrm{SCl}$. Solid line shows line of best fit; dotted lines shows 95\% confidence interval

while RD changes may correlate with the damage across the myelin sheath $[35,36]$. In the present study, we assessed the sequential FA, AD and RD values at the VWM of the rostro-caudal sector and found that the FA and AD values in the severe group were the lowest at the lesion epicenter at every time points. The FA and $\mathrm{AD}$ values did not always change in parallel. FA did not show any significant changes over time in both two severities of acute SCI. However, AD showed significant changes between day 3 and 7 . This characteristic might be due to the detectable axonal collapse at the early time points of acute SCI, which was consistent with previous research results $[37,38]$. The $R D$ values of the lesion epicenter in the severe group remained the highest but did not change with time and severities. Since the different parameters reflected different problems, it was necessary to combine multiple metrics for comprehensive analysis. A clinic study found that combination of DTI metrics could be used for differentiating malignant from benign compressed vertebrae as the malignant revealed lower MD and higher FA than benign involvement [31]. As to our work, the $\mathrm{FA}, \mathrm{AD}$, and $\mathrm{RD}$ values changed differently, so we needed to combine more detection methods to find out which value could reflect the damage of graded SCI over time more accurately.

MEP testing, which is regarded as an objective measure to quantifiably evaluate functional integrity of spinal pathways [39], is also a sensitive tool to detect the changes in conductivity of spinal cord. Because the principle of DTI and MEP measurements are related to the morphometry and permeability of the axonal membrane to water [20,21], we analyzed the correlation between the two methods, and found a parallel alteration in both MEP amplitudes and DTI measures (FA and AD). What's more, the current investigation showed significant correlations between acute (day 7 and 14) DTI metrics and acute BBB scores, as well as histopathology, which reflected the previous study in adults and rats $[40,41]$. Based on the above results, we concluded that $\mathrm{AD}$ value could accurately reflect the degree of spinal cord injury in the early acute phase, while FA seemed to show superiority in the subacute phase.

There are several limitations in our study. First, we preformed ex vivo in order to ensure high SNR and avoid the artifacts which would augment in vivo, but it is impossible to perform MRI scan on the same subject over time, and we will probably miss some details of the injury processes which will be obvious in vivo. In fact, although several studies have applied DTI to the acute SCI both in animal models as well as human subjects [11], limited by the significant artifact from respiratory and cerebrospinal fluid (CSF) motion, it still has difficulties in imaging and evaluation of the spinal cord [42], which needs further studies. We are going to find normative 
values of DTI metrics in vivo. Second, because of the injury, it is difficult to delineate the boundary of WM/GM, which will cause more variability in DTI metrics.

\section{Conclusions}

Using behavior evaluation, electrophysiological assessments, DTI and morphology, the present study examined the dynamic variation of acute $\mathrm{SCI}$ and explored the correlation between MEP and motor function in rats. It is very important to understand the pathophysiology and functional changes of acute SCI. Through such assessment, we may provide a useful information for the early identification of the severity of SCI and therefore give an optimal treatment.

\begin{abstract}
Abbreviations
DTI: Diffusion tensor imaging; WM: White matter; SCl: Spinal cord injury: MEPs: Motor evoked potentials; BBB: Basso: Beattie: and Bresnaha; FA: Fractional anisotropy; AD: Axial diffusivity; RD: Radial diffusivity; MRI: Magnetic resonance imaging; SD: Sprague-Dawley; BSA: Bovine serum albumin; ROls: Regions of interest; MD: Mean Diffusivity; SEM: Standard error of the mean; SD: Standard deviation; ANOVA: Analysis of variance; WWM: Ventrolateral white matter; DTT: Diffusion tensor tract fibers; MBP: Myelin Basic Protein; DPI: Days postinjury
\end{abstract}

\section{Acknowledgments}

We appreciate the labs of universities for providing us with the support and resources, which we need to be successful in our research endeavors.

\section{Authors' contributions}

$B C$ designed the study, prepared the animal ethics and conducted the study and participated in manuscript preparation and revision. QT helped in animal ethics, preparation and revision of the manuscript. WZ and QY helped in analysis of data in the BBB scores and MEPs studies. $\mathrm{HZ}$ participated in DTI mask drawings and MEPs studies. XL participated in the histology studies. FG participated in DTI acquisition and the analysis of the DTI metrics. HF and DJ both supervised the study, provided financial support, and contributed equally to this study. The authors read and approved the final manuscript.

\section{Funding}

This work was supported by the Major Innovation Project of Southwest Hospital (Grant number SWH2016ZDCX1011, SWH2017ZDCX1002) and Scientific Research Achievement Transformation Project of Chongqing Education Commission (41021300090243). The funding bodies are not responsible for the study design, data collection/analysis, decision of publication or preparation of the manuscript

\section{Availability of data and materials}

The datasets used and/or analysed during the current study are available from the corresponding author on reasonable request.

\section{Ethics approval and consent to participate}

Animal experimental procedures were performed under the Regulations for the Administration of Affairs Concerning Experimental Animals approved by the State Council of People's Republic of China. All experiments were conducted in accordance with animal care guidelines approved by the Laboratory Animal Welfare and Ethics Committee of Third Military Medical University (the Army Military Medical University).

\section{Consent for publication}

Not applicable.

\section{Competing interests}

The authors declare that there are no competing interests.

\section{Author details}

'Department of Orthopedics, The First Affiliated Hospital of Chongqing Medical University, Chongqing 400016, People's Republic of China.
${ }^{2}$ Department of Neurosurgery, Southwest Hospital, Third Military Medical University, Army Medical University, Chongqing 400038, People's Republic of China. ${ }^{3}$ Department of Radiology, West China Hospital, Sichuan University, Chengdu 610041, Sichuan, People's Republic of China. ${ }^{4}$ Department of Orthopedics, The Third Affiliated Hospital of Chongqing Medical University (Gener Hospital), Chongqing 401120, People's Republic of China.

Received: 28 March 2020 Accepted: 12 May 2020

Published online: 09 June 2020

\section{References}

1. Yiu G, He Z. Glial inhibition of CNS axon regeneration. Nat Rev Neurosci. 2006:7:617-27.

2. Kirshblum SC, Botticello AL, DeSipio GB, Fichtenbaum J, Shah A, Scelza W. Breaking the news: a pilot study on patient perspectives of discussing prognosis after traumatic spinal cord injury. J Spinal Cord Med. 2016;39:155-61.

3. Piazza M, Schuster J. Timing of surgery after spinal cord injury. Neurosurg Clin N Am. 2017;28:31-9.

4. Kirshblum SC, Burns SP, Biering-Sorensen F, Donovan W, Graves DE, Jha A, et al. International standards for neurological classification of spinal cord injury (revised 2011). J Spinal Cord Med. 2011;34:535-46.

5. Kirshblum SC, Waring W, Biering-Sorensen F, Burns SP, Johansen M, Schmidt-Read M, et al. Reference for the 2011 revision of the international standards for neurological classification of spinal cord injury. J Spinal Cord Med. 2011;34:547-54.

6. Ditunno JF, Little JW, Tessler A, Burns AS. Spinal shock revisited: a fourphase model. Spinal Cord. 2004;42:383-95.

7. Nishi RA, Liu H, Chu Y, Hamamura M, Su MY, Nalcioglu O, et al. Behavioral, histological, and ex vivo magnetic resonance imaging assessment of graded contusion spinal cord injury in mice. J Neurotrauma. 2007;24:674-89.

8. Medana IM, Esiri MM. Axonal damage: a key predictor of outcome in human CNS diseases. Brain. 2003;126:515-30.

9. Abdel Razek AAK. Routine and advanced diffusion imaging modules of the salivary glands. Neuroimaging Clin N Am. 2018;28:245-54.

10. Hulkower MB, Poliak DB, Rosenbaum SB, Zimmerman ME, Lipton ML. A decade of DTI in traumatic brain injury: 10 years and 100 articles later. AJNR Am J Neuroradiol. 2013;34:2064-74.

11. Kaushal M, Shabani S, Budde M, Kurpad S. Diffusion tensor imaging in acute spinal cord injury: a review of animal and human studies. J Neurotrauma. 2019;36:2279-86.

12. Razek A, El-Serougy L, Abdelsalam M, Gaballa G, Talaat M. Differentiation of residual/recurrent gliomas from postradiation necrosis with arterial spin labeling and diffusion tensor magnetic resonance imaging-derived metrics. Neuroradiology. 2018;60:169-77.

13. El-Serougy L, Abdel Razek AA, Ezzat A, Eldawoody H, El-Morsy A. Assessment of diffusion tensor imaging metrics in differentiating low-grade from high-grade gliomas. Neuroradiol J. 2016;29:400-7.

14. Steeves JD, Lammertse D, Curt A, Fawcett JW, Tuszynski MH, Ditunno JF, et al. Guidelines for the conduct of clinical trials for spinal cord injury (SCI) as developed by the ICCP panel: clinical trial outcome measures. Spinal Cord. 2007;45:206-21.

15. Shields CB, Ping Zhang Y, Shields LB, Burke DA, Glassman SD. Objective assessment of cervical spinal cord injury levels by transcranial magnetic motor-evoked potentials. Surg Neurol. 2006;66:475-83 discussion 83.

16. Glassman SD, Shields CB, Linden RD, Zhang YP, Nixon AR, Johnson JR. Anesthetic effects on motor evoked potentials in dogs. Spine. 1993;18:1083-9.

17. Hernandez-Lain A, Piedras MJ, Cavada C. Functional evaluation of paraplegic monkeys (Macaca mulatta) over fourteen months post-lesion. Neurosci Res. 2011;69:144-53.

18. Cao Q, Zhang YP, lannotti C, DeVries WH, Xu XM, Shields CB, et al. Functional and electrophysiological changes after graded traumatic spinal cord injury in adult rat. Exp Neurol. 2005;191(Suppl 1):S3-S16.

19. Garcia-Alias G, Verdu E, Fores J, Lopez-Vales R, Navarro X. Functional and electrophysiological characterization of photochemical graded spinal cord injury in the rat. J Neurotrauma. 2003;20:501-10.

20. Schwartz ED, Cooper ET, Fan Y, Jawad AF, Chin CL, Nissanov J, et al. MR diffusion coefficients in spinal cord correlate with axon morphometry. Neuroreport. 2005;16:73-6.

21. Clay JR. Axonal excitability revisited. Prog Biophys Mol Biol. 2005;88:59-90.

22. Noordzij M, Dekker FW, Zoccali C, Jager KJ. Sample size calculations. Nephron Clin Pract. 2011;118:c319-23. 
23. Yuan J, Liu W, Zhu H, Chen Y, Zhang X, Li L, et al. Curcumin inhibits glial scar formation by suppressing astrocyte-induced inflammation and fibrosis in vitro and in vivo. Brain Res. 2017;1655:90-103.

24. Plemel JR, Duncan G, Chen KW, Shannon C, Park S, Sparling JS, et al. A graded forceps crush spinal cord injury model in mice. J Neurotrauma. 2008:25:350-70.

25. Basso DM, Beattie MS, Bresnahan JC. A sensitive and reliable locomotor rating scale for open field testing in rats. J Neurotrauma. 1995;12:1-21.

26. Xia M, Chen W, Wang J, Yin Y, Guo C, Li C, et al. TRPA1 activation-induced myelin degradation plays a key role in motor dysfunction after Intracerebral hemorrhage. Front Mol Neurosci. 2019;12:98.

27. Redondo-Castro E, Navarro X, Garcia-Alias G. Longitudinal evaluation of residual cortical and subcortical motor evoked potentials in spinal cord injured rats. J Neurotrauma. 2016;33:907-16.

28. Wang C, Song L, Zhang R, Gao F. Impact of fixation, coil, and number of excitations on diffusion tensor imaging of rat brains at 7.0 T. Eur Radiol Exp. 2018;2:25.

29. Ellingson BM, Salamon N, Holly LT. Imaging techniques in spinal cord injury. World Neurosurg. 2014;82:1351-8.

30. Razek A, Ashmalla GA. Assessment of paraspinal neurogenic tumors with diffusion-weighted MR imaging. Eur Spine J. 2018;27:841-6.

31. Razek A, Sherif FM. Diagnostic accuracy of diffusion tensor imaging in differentiating malignant from benign compressed vertebrae. Neuroradiology. 2019;61:1291-6.

32. Cheran S, Shanmuganathan K, Zhuo J, Mirvis SE, Aarabi B, Alexander MT, et al. Correlation of MR diffusion tensor imaging parameters with ASIA motor scores in hemorrhagic and nonhemorrhagic acute spinal cord injury. J Neurotrauma. 2011;28:1881-92.

33. Mukherjee P. Diffusion tensor imaging and fiber tractography in acute stroke. Neuroimaging Clin N Am. 2005;15:655-65 xii.

34. Kim JH, Song SK, Burke DA, Magnuson DS. Comprehensive locomotor outcomes correlate to hyperacute diffusion tensor measures after spinal cord injury in the adult rat. Exp Neurol. 2012;235:188-96.

35. Tu TW, Kim JH, Yin FQ, Jakeman LB, Song SK. The impact of myelination on axon sparing and locomotor function recovery in spinal cord injury assessed using diffusion tensor imaging. NMR Biomed. 2013;26:1484-95.

36. Kim JH, Loy DN, Liang HF, Trinkaus K, Schmidt RE, Song SK. Noninvasive diffusion tensor imaging of evolving white matter pathology in a mouse model of acute spinal cord injury. Magn Reson Med. 2007;58:253-60.

37. Yung A, Mattucci S, Bohnet B, Liu J, Fournier C, Tetzlaff W, et al. Diffusion tensor imaging shows mechanism-specific differences in injury pattern and progression in rat models of acute spinal cord injury. Neuroimage. 2019;186:43-55.

38. Song W, Song G, Zhao C, Li X, Pei X, Zhao W, et al. Testing pathological variation of white matter tract in adult rats after severe spinal cord injury with MRI. Biomed Res Int. 2018:2018:4068156.

39. Curt A, Keck ME, Dietz V. Functional outcome following spinal cord injury: significance of motor-evoked potentials and ASIA scores. Arch Phys Med Rehabil. 1998;79:81-6.

40. Petersen JA, Wilm BJ, von Meyenburg J, Schubert M, Seifert B, Najafi Y, et al, Chronic cervical spinal cord injury: DTI correlates with clinical and electrophysiological measures. J Neurotrauma. 2012;29:1556-66.

41. Zhao C, Rao JS, Pei XJ, Lei JF, Wang ZJ, Yang ZY, et al. Longitudinal study on diffusion tensor imaging and diffusion tensor tractography following spinal cord contusion injury in rats. Neuroradiology. 2016;58:607-14.

42. Krishna V, Andrews H, Varma A, Mintzer J, Kindy MS, Guest J. Spinal cord injury: how can we improve the classification and quantification of its severity and prognosis? J Neurotrauma. 2014;31:215-27.

\section{Publisher's Note}

Springer Nature remains neutral with regard to jurisdictional claims in published maps and institutional affiliations.

Ready to submit your research? Choose BMC and benefit from:

- fast, convenient online submission

- thorough peer review by experienced researchers in your field

- rapid publication on acceptance

- support for research data, including large and complex data types

- gold Open Access which fosters wider collaboration and increased citations

- maximum visibility for your research: over $100 \mathrm{M}$ website views per year

At BMC, research is always in progress.

Learn more biomedcentral.com/submissions 